\title{
A PROSPECÇÃO DO FUTURO COMO SUPORTE À BUSCA DE INFORMAÇÕES PARA A DECISÃO EMPRESARIAL
}

\section{PROSPECT OF FUTURE AS A SUPPORT TO SEARCH FOR INFORMATION FOR BUSINESS DECISION}

\section{UNA PERSPECTIVA DE FUTURO COMO APOYO PARA BUSCAR INFORMACIÓN} PARA LA TOMA DE NEGOCIOS

\section{Nelson Daishiro Yoshida}

Doutorando do Programa de Pós Graduação em Administração da Faculdade de Economia, Administração e Contabilidade da Universidade de São Paulo - PPGA FEA/USP

E-mail: nelson.yoshida@uol.com.br (Brasil)

\section{James Terence Coulter Wright}

Doutor pela Universidade de São Paulo - USP

Líder de grupo de pesquisa CNPq

E-mail: jtwright@usp.br (Brasil)

\section{Renata Giovinazzo Spers}

Pós-doutorado na Universidade Vanderbilt, EUA

Líder do grupo de pesquisas Programa de Estudos do Futuro da Universidade de São Paulo PROFUTURO/USP

E-mail: renatag@usp.br (Brasil) 
A PROSPECÇÃO DO FUTURO COMO SUPORTE À BUSCA DE INFORMAÇÕES PARA A DECISÃO EMPRESARIAL

\title{
RESUMO
}

Nesta pesquisa foi estudada a prática de prospecção do futuro como suporte à busca de informações para a decisão. Dentre os objetivos da pesquisa estão a busca de associações entre os métodos de prospecção e temas estudados, a importância atribuída à atividade, a frequência de uso, os horizontes de tempo e o grau de satisfação com os resultados obtidos. A pesquisa é realizada por metodologia mista, foram feitos levantamento de dados quantitativos e estudos de casos. Foi identificado que há associação entre a categoria da informação prospectada e os métodos. É atribuída importância para a atividade, dependendo do tipo de decisão ser estratégico ou tático, e este fator também influencia a frequência em que a atividade é praticada. O horizonte de tempo da prospecção está associado aos métodos. Os métodos mais praticados são os que apresentam a possibilidade de interatividade pessoal, sendo que métodos objetivos, como os que utilizam equações, não figuraram entre os mais praticados. Nos casos estudados foi identificado que os métodos mais praticados são baseados em opinião e julgamento e utilizam a interatividade pessoal fortemente. Há preocupação manifestada sobre a necessidade de estruturação da atividade nas empresas e sobre a prática da mesma seguindo recomendações metodológicas.

Palavras-chave: Estratégia; Estudos do Futuro; Tomada de Decisão.

\section{PROSPECT OF FUTURE AS A SUPPORT TO SEARCH FOR INFORMATION FOR BUSINESS DECISION}

\begin{abstract}
The use of future studies methodologies to provide information for decision making has been studied in this research. The objectives of the research are to identify the association of methods to different information categories, the importance of future studies, the frequency of use, the time horizon and users perceived satisfaction with the results. The research is implemented through a mixed-methods methodology, with a survey and case studies. Combined results have been analyzed in a complementary approach. Results fromthe sample indicate that there is an association of methods with information categories. Evaluation of the importance of future studies is high and depends on the decision type to be strategic or tactical,it also influences the frequency of use. Time horizon is associated to methods. The most used methods are the ones with high personal interaction, on the other hand, objective methods, e.g. mathematical techniques, are not among the most used. In the case studies results, methods based on judgment and opinion with high personal interaction are the most used. There were concerns about the need for more structure to deal with future studies in the company and also about its practice in accordance with methodological recommendations.
\end{abstract}

Keywords: Strategy; Futures Studies; Decision Making. 


\title{
UNA PERSPECTIVA DE FUTURO COMO APOYO PARA BUSCAR INFORMACIÓN
}

\author{
PARA LA TOMA DE NEGOCIOS
}

\section{RESUMEN}

En esta investigación se estudió la práctica de la prospección del futuro para apoyar la búsqueda de información para la decisión. Entre los objetivos de la investigación son la búsqueda de asociaciones entre los métodos de prospección y sujetos estudiados, la importancia atribuida a la actividad, frecuencia de uso, los horizontes de tiempo y el grado de satisfacción con los resultados. La encuesta se lleva a cabo mediante una metodología mixta se hicieron datos de encuestas cuantitativas y estudios de casos. Se identificó que existe una asociación entre la categoría de información prospectado y métodos. Importancia se atribuye a la actividad, dependiendo del tipo de decisión de ser estratégica o táctica, y este factor también influye en la frecuencia a la que se practica la actividad. El horizonte temporal se asocia con los métodos de prospección. Los métodos practicados son los que tienen la posibilidad de interacción personal y métodos objetivos, tales como los que utilizan ecuaciones, se encontraban entre los más populares. En los casos estudiados se identificó que los métodos más practicados se basan en la opinión y el juicio y el uso de la interactividad personal fuerte. Existe la preocupación expresada acerca de la necesidad de estructurar la actividad en los negocios y en la práctica de seguir las mismas recomendaciones metodológicas.

Palabras-clave: Estrategia; Los Futuros Estudios; Toma de Decisiones. 


\section{INTRODUÇÃO}

A tomada de decisão é frequentemente associada a incertezas em relação ao futuro. A limitação quanto à disponibilidade de informações que permitam analisar o futuro e suas influências sobre a decisão tomada no presente representam um desafio para gestores.

A hipótese de que o futuro seja passível de antecipação é irreal, pois o futuro é imprevisível. Por outro lado, gestores são responsáveis pelas suas decisões, e estas últimas necessitam de premissas e análises sobre o futuro, então seria igualmente irreal crer que os gestores não recorram a métodos de prospecção. Devido à complexidade do ambiente atual e a existência de múltiplos stakeholders interessados nas organizações e instituições, tomar decisões deixando de prospectar o futuro pode representar um risco maior do que o risco de prospectar e errar.

Dessa forma, o presente estudo tem como problema de pesquisa o estudo da prática de prospecção do futuro como suporte à busca de informações para a tomada de decisão. A pergunta de pesquisa central é - A prospecção é praticada? A partir dela, são propostas outras questões decorrentes: Que temas induzem à prospecção? Esses temas estão associados com os métodos de prospecção? A atividade é importante? Qual a frequência em que é praticada? Qual o horizonte de tempo prospectado? O tipo de decisão envolvida influencia a prática?

\subsection{OBJETIVOS}

Para elucidar o problema de pesquisa proposto, esta pesquisa tem como objetivos: (1)estudar a prática da prospecção do futuro e a importância atribuída à atividade pelos gestores nas empresas; (2) estudaraassociaçãoentreosmétodosdeestudosdofuturoeascategoriasde informações que são prospectadas; (3) verificar a satisfação com os resultados obtidos e, por fim, (4) identificara influência dos tipos de decisão, estratégico e tático, sobre a prospecção do futuro.

\section{REFERENCIAL TEÓRICO}

A revisão dos conceitos de prospecção do futuro desta pesquisa é realizada considerando os objetivos acima mencionados. São incluídos conceitos sobre a prática de prospecção, a teoria que trata da prospecção e da tomada de decisão, os métodos de prospecção e as variáveis relacionadas ao seu uso e as categorias de informação prospectadas. 


\subsection{A PRÁTICA DA PROSPECÇÃO E SEUS FATORES RELEVANTES}

O estudo do futuro não deve buscar a certeza, uma vez que ela não pode ser alcançada (Sardar, 2010). O foco demasiadamente concentrado também pode levar a resultados limitados (Day \& Schoemaker, 2004).

Schwarz (2008) argumenta que gestores compreendem a necessidade de se estudar o futuro. Dentre os métodos que ganharão importância ao longo do tempo, estãoaqueles que abrangem análises de contexto, de monitoramento de tendências do ambiente e o de formulação de cenários. $\mathrm{O}$ maior desafio é a utilização dos métodos nas empresas, pois os gestores não sentem falta de métodos e técnicas disponíveis, contudo, sentem dificuldade na aplicação dos mesmos (Schwarz, 2008).

A escolha do método (ou da combinação de métodos) deve ser feita considerando alguns itens, dentre eles horizonte de tempo, a urgência na disponibilidade da informação, a frequência de atualizações das informações, o conhecimento dos métodos, a disponibilidade e capacidade de processamento computacional e o orçamento disponível. (Georgoff \& Murdick, 1986).

Temas que sempre devem estar presentes na prática de prospecção são: objetivos,horizonte de tempo, tipos de fontes de dados, familiaridade com os métodos, critérios de escolha do método, revisões de julgamento subjetivo e comunicação de resultados, conforme Winklhofer, Diamantopoulos e Witt (1996).

A falta de familiaridade, de conhecimento técnico ou de experiência na aplicação dos métodos pode ser um obstáculo (Hogarth \& Makridakis, 1981; Dawes, 1979). Mentzler e Kahn (1995) estudaram as relações entre a familiaridade com os métodos e a satisfação com os resultados, sendo que a pesquisa destes autores foi longitudinal entre 1985 e 1994. Os métodos mais utilizados foram opinião de executivos, pesquisas de mercado sobre expectativas de clientes, regressão e linhas de tendência.

Mahmoud (1989) contribuiu propondo a combinação de métodos de prospecção. A proposta abrange métodos de naturezas diferentes, como os quantitativos com os de julgamento. Godet e Roubelat (1996) indicavam formas de tratar incertezas de cenários por meio do uso combinado de métodos como Delphi e análise morfológica.

Considerando os argumentos dos autores citados, observa-se que a prática dos métodos de prospecção deve considerar fatores relacionados com o tema em análise e a expectativa de tempo e frequência em que resultados são necessários. Dessa forma, nesta pesquisa são consideradas como variáveis relativas aos métodos de prospecção: Importância da atividade, Horizonte de tempo, Frequência de Uso e Satisfação com os resultados. 


\subsection{A PROSPECÇÃo DO FUTURO, TOMADA DE DECISÃo, INCERTEZA E INFORMAÇÃO}

A tomada de decisões é uma atividade caracterizada por diversas influências. O jogo político, o uso do poder e o controle de informações influenciam as escolhas a serem consideradas (Eisenhardt \& Zbaracki, 1992). Galbraith (1974) argumentou que quanto mais incertezas houver, maior é o volume de informação processada, e Phlips (1988) argumenta que a informação tem papel importante na decisão.

As incertezas não são relacionadas somente ao estado do ambiente. Devem ser analisadas também as incertezas relacionadas aos efeitos do ambiente sobre a organização, bem como as possíveis respostas que podem ser adotadas pela organização (Milliken, 1987).

A motivação para se estudar o futuro é a possibilidade de poder se preparar para atingir objetivos escolhidos ou prevenir e preparar a organização para situações futuras. A preparação para o futuro é realizada por um planejamento estabelecido por decisões orientadas para o futuro (Hogarth \& Makridakis, 1981).

No processo de tomada de decisão, o ser humano é eficaz apenas em relação à seleção e codificação da informação para subsidiar a decisão. Em se tratando de integrar as informações de forma a permitira tomada de decisão, a limitação humana prejudica o processo (Dawes, 1979).

Há situações em que o uso de métodos quantitativos de estudos do futuro pode ser mais recomendado do que os de julgamento (Georgoff \& Murdick, 1986). Em contraste, estudar o futuro inclui julgamentos subjetivos, mesmo nos casos em que a decisão seja baseada utilizando-se métodos quantitativos. A literatura indica haver alterações nos resultados em parte significativa dos casos, ou seja, os gestores julgaram subjetivamente o valor dos resultados obtidos, alterando-os posteriormente (Fildes \& Goodwin, 2007).

É comum a tendência de inserção de vieses na avaliação das chances de um determinado evento ocorrer, em função do maior ou menor desejo de que aquele evento ocorra de fato (Bolger\&Wright, 1994).

Por um lado admitir erros na prospecção pode significar que se assume o tratamento das incertezas como possivelmente improdutivo; por outro lado admitir que esteja correto significa assumir a própria ilusão de controle, que é um viés cognitivo. Erros de utilização dos métodos prospectivos podem ser oriundos das dificuldades do ser humano na tomada de decisões sob condições de incertezas, argumentam Makridakis, Hogarth e Gaba (2009).

O ser humano não consegue gerar todas as alternativas de ações plausíveis para uma decisão, também não é capaz de coletar e processar todas as informações para prever as 
consequências das alternativas, e finalmente, mesmo que as consequências pudessem ser antecipadas, o ser humano não consegue calcular com precisão o valor delas (Simon, 1997).

Um componente relacionado ao uso de métodos prospectivos é a necessidade de análises preliminares, de premissas e de fontes de informações a serem utilizadas na prospecção. O processo de utilizar (praticar) os métodos é tão importante quanto o uso dos resultados obtidos (Strand, 1999).

A informação e as análises realizadas a partir de sua disponibilidade têm papel importante na tomada de decisão. Quando ela trata sobre o futuro, é certo que estará acompanhada de incertezas que necessitam ser tratadas. Dessa forma, a variável Informação se constitui um foco desta pesquisa.

\subsection{TEMAS INDUTORES SOBRE O FUTURO: CATEGORIAS DE INFORMAÇÕES}

A abordagem para agregar os temas que induzem à prospecção, as categorias de informação para esta pesquisa, se inicia por meio da análise PESTEL (sigla do original em inglês), que agrupa as informações em fatores políticos $(\mathrm{P})$, econômicos $(\mathrm{E})$, sociais $(\mathrm{S})$, tecnológicos $(\mathrm{T})$, meio ambiente (E) e legais (L). Walsh (2005) combinou esta técnica com a de análise de recursos e o método de cenários para pesquisar sobre o desempenho de empresas em lidar com incertezas relacionadas às mudanças no meio ambiente. Kaplan e Norton (2008) indicam a análise PESTEL como modelo para subsidiar a análise estratégica.

Porter et al. (1991) argumentam que temas como os recursos naturais, matéria-prima, capital financeiro, recursos humanos e infraestrutura são relevantes para a organização de base tecnológica. Baseado nesses argumentos adota-se nesta pesquisa a categoria Insumos e Recursos como um tema passível de prospecção.

Schoemaker (1995) argumenta que gestores nas organizações podem não perceber ou não gerar novas oportunidades de negócios para a organização.Walsh (2005) também propõe que os estudos que buscam relações entre a estratégia, o ambiente e o desempenho de organizações precisam considerar variáveis sobre o foco do negócio.Dessa forma, o tema Oportunidades de Negócios é adotado como tema de prospecção.

O estudo de Walsh (2005) tinha foco nos atributos do produto. Considerando que um produto (ou serviço) tem muitos atributos e contém diversos componentes tecnológicos já disponíveis ou ainda por serem desenvolvidos, então é recomendável que Produto passe a ser considerado como um tema de prospecção. 
A Prospecção do Futuro como Suporte à Busca de Informações para a Decisão Empresarial

O ambiente demográfico é um fator relacionado ao mercado que representa importante influência na estratégia. Variáveis como taxa de crescimento, grau de instrução e perfil de renda tem sua relevância na estratégia de marketing, conforme Kotler e Keller (2006). Dessa forma, nesta pesquisa é adotado o tema Mercado como objeto de prospecção.

Em resumo, os temas indutores da prospecção, as categorias da variável Informação desta

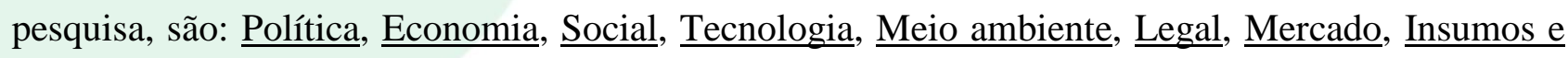
$\underline{\text { Recursos, Produtos (incluindo serviços) e Oportunidades de Negócios. }}$

\subsection{MÉTODOS DE PROSPECÇÃO}

Esta pesquisa inclui os métodos de prospecção a seguir:

\section{a. Análise Bibliométrica}

A análise bibliométrica é um método de contagem de termos sobre conteúdos bibliográficos. Segundo Daim, Ploykitikoon, Kennedy, e Choothian (2008)a técnica possibilita o auxílio no processo de tomada de decisões, pois permite explorar, organizar e analisar grandes massas de dados.

A identificação do número de vezes em que termos são citados, e a taxa de crescimento desse número, indicaria o nível de atividade de pesquisa sobre um tema em prospecção. As aplicações típicas são para rastreamento de tendências de pesquisas ou identificação de tecnologias emergentes, conforme Vidican, Woon e Madnick (2009) e Porter (2007).

\section{b. Cenários}

O método de cenários consiste em construir, de forma criativa, estruturada e disciplinada, narrativa sobre possíveis contextos futuros aplicáveis para análise estratégica das organizações (Schoemaker, 1995). O método não é aplicado para encontrar um acerto sobre previsões de como será o futuro. Ele visa, principalmente, agregar mais informações ao processo, melhorar as informações e assim compreender as decisões envolvidas para que estas sejam tomadas observando o que tenha sido prospectado, e assim atingir objetivos relacionados ao futuro (Wright \& Spers, 2006).

Cenários tanto pode ter uma abordagem qualitativa, na qual se enfatiza o poder analítico e criativo das pessoas, quanto pode ter uma abordagem quantitativa na qual são realizadas simulações computacionais com o uso de probabilidades e valores atribuídos para as variáveis consideradas nos modelos de cenários (Chermack, 2006). 


\section{c. Delphi}

A técnica Delphi passou a ser difundida a partir dos anos 1960, quando os pesquisadores Olaf Helmer e Norman Dalker, da Rand Corporation, coordenaram estudo para aprimorar o uso de consulta a especialistas. $\mathrm{O}$ aprimoramento proposto pelos pesquisadores era centrado em três condições, conforme Martino (1993, p.17-19): (1) anonimato dos especialistas respondentes, (2) representação estatística da distribuição dos resultados obtidos e (3) feedback aos respondentes com o intuito de possibilitar a reavaliação de suas respostas dadas em etapa anterior, o que cria condições para convergência de respostas. O conceito básico consiste em consultar um grupo de especialistas sobre determinado tema e seus possíveis estados futuros. A consulta é realizada por meio de questionário, aplicado em mais de uma rodada de consulta com o objetivo de se obter uma convergência das respostas. A consulta preserva o anonimato dos respondentes, visando evitar o alinhamento das opiniões com as respostas de algum especialista de destacada reputação. A cada rodada é realizado um feedback aos respondentes do grupo de especialistas, e é implementada uma nova consulta que permite ao respondente calibrar seu julgamento (Wright \& Giovinazzo, 2000).Estudos empíricos mostram que o método gera resultados mais acurados em comparação aos demais métodos de grupos de interação não estruturados (Rowe \& Wright, 1999).

\section{d. Métodos matemáticos}

Georgoff e Murdick (1986) classificam métodos que relacionam variáveis entre si como de “associação ou de causa", pois eles têm foco em possibilitar a associação entre variáveis ou a identificação de relação de causa entre elas. Nesta pesquisa os métodos matemáticos incluem: Correlação, regressão, modelos econométricos, extrapolação de dados históricos.

\section{e. Palestras de especialistas}

A palestra de especialistas é um método que é utilizado de forma combinada com outros métodos, muitas vezes com aqueles que utilizam o julgamento e a opinião de pessoas. Georgoff e Murdick (1986) classificam os métodos que utilizam interação pessoal como baseados em julgamento e opinião de pessoas. A interação entre pessoas permite explorar diversas opiniões e sintetizá-las para poder representar o futuro. O método de palestras de especialistas é bem utilizado porque ele permite questionar, esclarecer dúvidas e debater sobre as informações com o especialista presente. 
A Prospecção do Futuro como Suporte à Busca de Informações para a Decisão Empresarial

\section{f. Pesquisas de mercado}

Nesta pesquisa são os métodos que utilizam estimativas de contagem, de propensão a consumo ou uso de determinado produto ou serviço em distintos mercados segmentados por algum critério de interesse do pesquisador. Este método está classificado por Georgoff e Murdick (1986) como “de contagem”, pois busca traduzir em números o potencial futuro de demanda, de vendas ou uso de produto ou serviço. A pesquisa de mercado permite uma delimitação clara como um método de prospecção, ao possibilitar um meio pelo qual o planejamento de marketing pode ser realizado (Kotler \& Keller, 2006, p.98).

\section{g. Previsões e projeções de executivos, força de vendas e distribuidores}

São métodos em que se utilizam previsões de pessoas. Esses métodos, quando aplicados em horizontes de tempo mais curtos, podem ser relacionados dentre os descritos a seguir (Georgoff \& Murdick, 1986): previsões e projeções de força de vendas, de distribuidores e fornecedores, previsões e projeções de executivos, palpite simples e analogia histórica. A estimativa de demanda futura é a aplicação mais comum desses métodos. Análises de intenção de compra, composição das opiniões de forças de vendas, consulta aos especialistas e análise das vendas acumuladas são técnicas utilizadas para complementar o método de previsão e projeção de executivos.

\section{h. Roadmap tecnológico}

Uma descrição que pode ser utilizada para este método é: o mapeamento de tecnologia por meio de uma visão gráfica, ao longo do tempo, das relações entre diversos componentes relacionados com tecnologias, com os produtos que as incorporam, com as áreas da organização que as aplicam e as utilizam e finalmente com os mercados consumidores. $\mathrm{O}$ método permite aprimorar a visão do ambiente futuro das tecnologias. Contribui também para o desenvolvimento de conhecimento acerca da tecnologia, em outras palavras, aspectos como o que ela é, por que ela pode ser adotada, por quem, quando, como e onde será adotada, conforme Phaal, Farrukh e Probert (2004). Roadmap Tecnológico tem a ver com tecnologia e produto, e também com a rota a ser percorrida para se chegar ao produto, com as relações entre os diversos componentes de tecnologia, chegando ao mapa de como poderá chegar ao futuro (Lee \& Park, 2005).A viabilização de algumas tecnologias componentes pode depender da disponibilidade de outras tecnologias que as precedem. Dessa forma o Roadmap Tecnológico contribui para analisar informações sobre como pode ocorrer o 
encadeamento temporal dessas tecnologias e, ao final, a disponibilidade do produto ou serviço resultante (PHAAL et al., 2004).

\section{PROCEDIMENTOS METODOLÓGICOS}

A metodologia adotada nesta pesquisa é orientada para explorar e entender o fenômeno de forma preliminar. Portanto é adequado um estudo exploratório e descritivo, segundo Sampieri, Collado e Lucio (2006). A metodologia utilizada é mista, ou seja, com mais de um método de pesquisa, visando aumentar o potencial de análise (Creswell, 2010). Por decorrência disso, o presente estudo é desenvolvido por meio da combinação de método quantitativo com qualitativo, buscando sinergias entre os métodos (Eisenhardt, 1989).

A coleta de dados é realizada por dois meios: (1) questionário em forma de levantamento (survey) para a parte quantitativa e (2) estudos de casos por entrevista pessoal para a parte qualitativa. Em termos de amostragem, para esta pesquisa optou-se por uma escolha dirigida por meio de amostragem não probabilística. A base de respondentes utilizada é formada pelos alunos e ex-alunos do MBA Executivo Internacional da Fundação Instituto de Administração (FIA), caracterizando-se, portanto, como uma amostra por conveniência.

O questionário foi aplicado para 1.324 executivos de empresas, com contatos disponíveis,sendoque127respondentes concluíram integralmente o preenchimento do questionário da pesquisa.

As entrevistas da parte qualitativa foram realizadas com gestores de quatro organizações: (1) NEC Brasil, (2) Bioagência, (3) Centro de Tecnologia Canavieira (CTC) e (4) Itaipu Binacional. Os critérios para escolha dessas empresas foram quatro: (1) alto dinamismo do setor (NEC Brasil telecom), (2) atuação em setor de liderança tecnológica (Bioagência - etanol de cana de açúcar), (3) centro de pesquisa do setor de liderança tecnológica (CTC - etanol) e (4) setor de grande longevidade da operação (Itaipu - geração de energia hidrelétrica). Os relatos foram utilizados para complementar as análises da pesquisa quantitativa.

O Quadro 1a seguir mostra o modelo conceitual teórico e a matriz metodológica adotada neste estudo. 
A Prospecção do Futuro como Suporte à Busca de Informações para a Decisão Empresarial

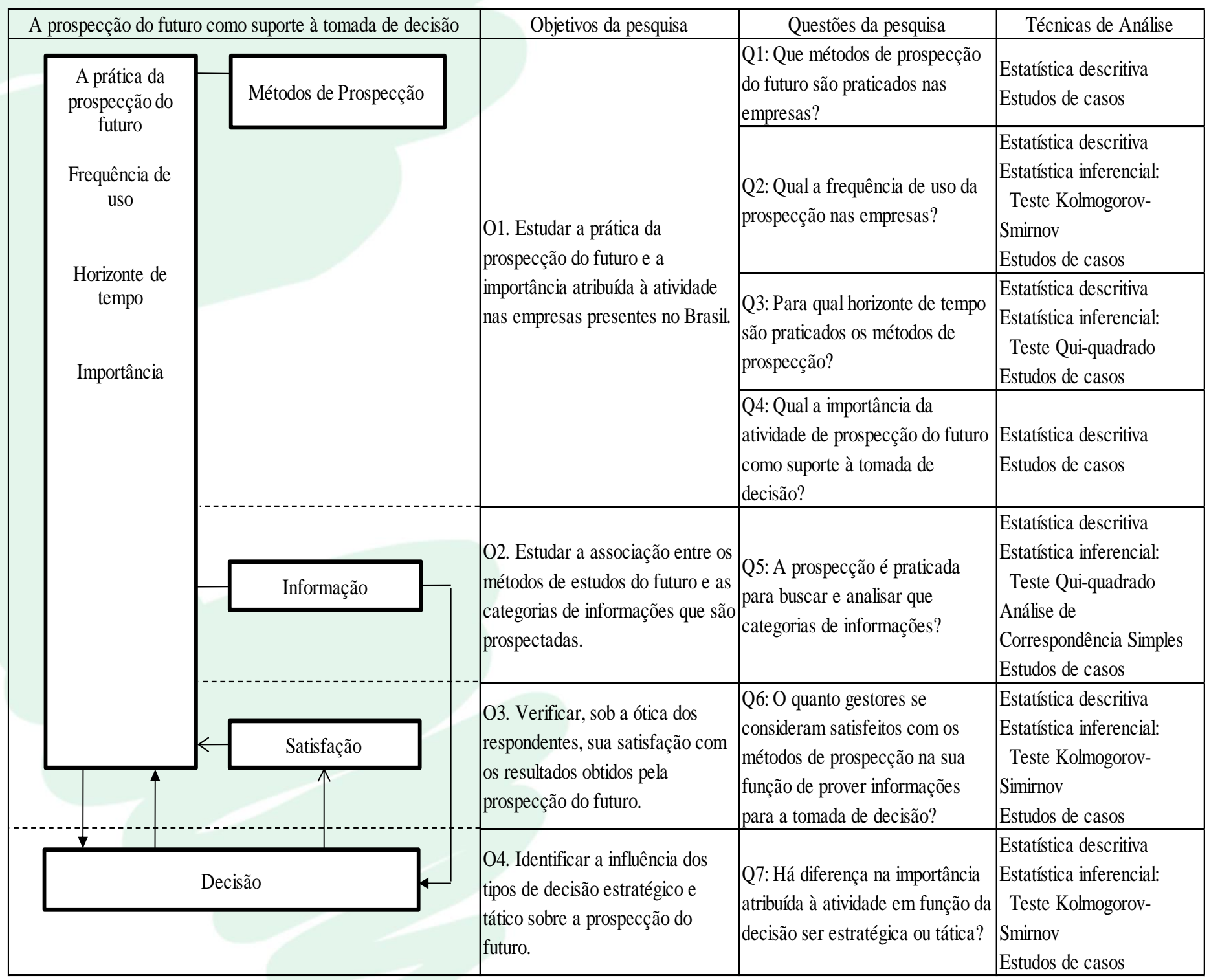

Quadro 1 - Modelo Conceitual Teórico e matriz metodológica Fonte: Elaborado pelos autores.

A pesquisa foi executada durante o primeiro semestre de 2011.

\section{ANÁLISE E DISCUSSÃO DE RESULTADOS}

\subsection{SOBRE A PRÁTICA DE MÉTODOS DE PROSPECÇÃO}

Neste item se analisou a prática da prospecção do futuro nas organizações, e os resultados quantitativos mostraram que os métodos mais utilizados são aqueles que permitem interação entre pessoas: Na ordem: Cenários, Pesquisas de Mercado, Previsões e Projeções de Executivos, Força de Vendas e Distribuidores e Palestras de Especialistas, conforme mostra a Tabela 1. 
Nelson Daishiro Yoshida, James Terence Coulter Wright \& Renata Giovinazzo Spers

Tabela 1 - Métodos de prospecção do futuro praticados

\begin{tabular}{|c|c|c|}
\hline MÉTODOS UTILIZADOS & FREQUÊNCIA & $\%(100 \%=127)$ \\
\hline Cenários & 108 & $85 \%$ \\
\hline Pesquisa de mercado & 95 & $75 \%$ \\
\hline Previsões e projeções de executivos, força de vendas & 93 & $73 \%$ \\
\hline Palestras de especialistas & 70 & $55 \%$ \\
\hline Métodos matemáticos/estatísticos & 65 & $51 \%$ \\
\hline Roadmap tecnológico & 44 & $35 \%$ \\
\hline Método Delphi & 15 & $12 \%$ \\
\hline Análise bibliométrica & 9 & $7 \%$ \\
\hline Nenhuma das anteriores (a prospecção não é praticada) & 3 & $2 \%$ \\
\hline Outros & 10 & $8 \%$ \\
\hline Total & 512 & \\
\hline
\end{tabular}

Fonte: Elaborado pelos autores.

Nos casos estudados, observou-se que a possibilidade de interagir pessoalmente com outras pessoas durante a prospecção é um facilitador no processo. A confiança em métodos que utilizam modelos ou técnicas instrumentalizadas e que geram resultados sem julgamento humano (sem interação ou debate) não é elevada, o que converge com o estudo de Fildes e Goodwin (2007).

Em nível intermediário de respostas encontram-se os métodos Matemáticos com $51 \%$. Este resultado mostra que aplicações típicas que são atendidas pelo uso de ferramentas já consolidadas, inclusive com softwares de apoio, ainda não são utilizadas de forma disseminada. Em 1979, Dawes já indicava esse fenômeno, e mesmo mais de trinta anos depois ele ainda ocorre.

As entrevistas realizadas também indicaram a ocorrência desse fenômeno. O caso do CTC ilustra a situação. Naquele centro de pesquisa há certa preferência por métodos e técnicas 
prescritivas para prospecção. Por outro lado, nem sempre isso é possível, uma vez que alternativas em análise impactam diferentes partes na cadeia da cana de açúcar, e as pessoas que representam estas partes influenciam nos critérios de análise. Trata-se de uma questão de agentes de influência na decisão. Evitar essas influências é impossível, segundo o relato obtido. Ao final, a ocorrência de julgamentos sobre os dados obtidos por modelos instrumentalizados, como os matemáticos, por exemplo, é um fenômeno corriqueiro.

\subsection{SOBRE A FREQUÊNCIA DE USO DA PROSPECÇÃO DO FUTURO}

Observa-se que há diferença na frequência de uso da prospecção para decisões táticas e estratégicas, ou seja, os resultados para a frequência de uso da prospecção mostraram que o tipo de decisão influencia a frequência de uso, $\chi 2=33,469, \mathrm{p}<0,05$, conforme Gráfico 1 .

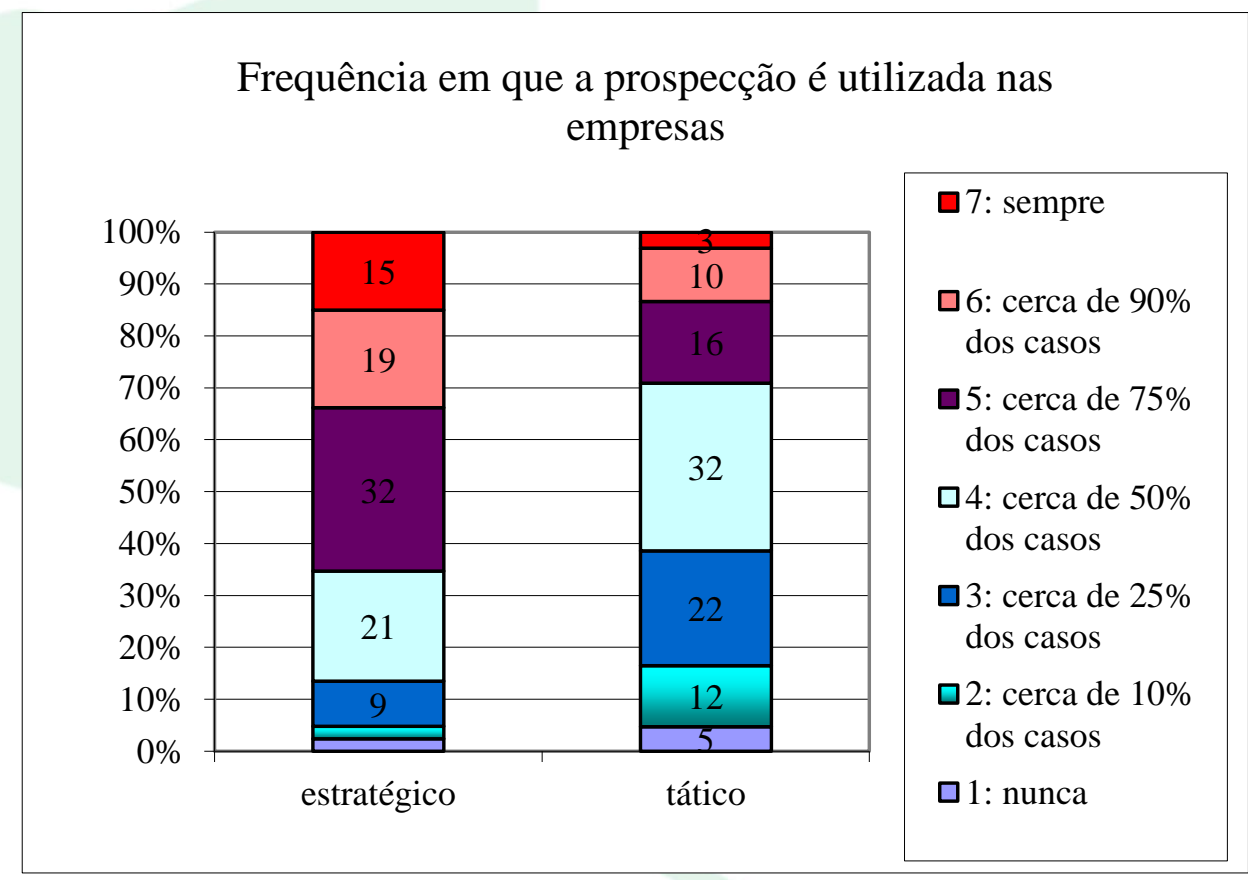

Gráfico 1 - Frequência de uso da prospecção do futuro

Fonte: Elaborado pelos autores.

Nos casos estudados identificou-se que para decisões táticas o horizonte de tempo é menor do que nas estratégicas, e a responsabilidade do gestor é mais direta, pois impacta os resultados do orçamento. Dessa forma, a atuação é mais focada no monitoramento das variáveis, que já foram identificadas em etapas de prospecção anteriores. Por decorrência disso, a frequência desta última atividade é menor. No caso da decisão estratégica, há muitas incertezas sobre quais variáveis devem ser prospectadas, por consequência, a prospecção é mais frequente nesses casos. 
Nelson Daishiro Yoshida, James Terence Coulter Wright \& Renata Giovinazzo Spers

\subsection{SOBRE OS MÉTODOS DE PROSPECÇÃO E O HORIZONTE DE TEMPO}

Com relação ao horizonte de tempo da prospecção, somando-se as frequências para “próximo trimestre” e "até 6 meses” e testando-se pela prova de Qui-Quadrado, tem-se:

$$
\chi 2=130,8, p=0,000<0,05
$$

Isto mostra evidências de que Método de Prospecção e Horizonte de Tempo não são variáveis independentes. Cenários e Roadmap Tecnológico apresentam maior horizonte de tempo na amostra desta pesquisa. Cerca de $50 \%$ dos respondentes atribuíram no mínimo 5 anos ou mais.Métodos Matemáticos e Método Delphi resultaram num patamar intermediário em termos de horizonte de tempo com $37 \%$ e $31 \%$ dos respondentes atribuindo mínimo de 5 anos, respectivamente.

Entre os utilizadores de Análise Bibliométrica, Previsões e Projeções de Executivos, Palestras de Especialistas e Pesquisas de Mercado, o horizonte de tempo mais citado foi de até um ano, portanto para curto prazo. Com exceção do método de Cenários, os métodos utilizados para horizontes de tempo mais longos não são muito utilizados.

Nos casos estudados foi constatado que o curto prazo mobiliza os gestores das empresas pela pressão do orçamento e das metas, portanto há maior envolvimento e engajamento na atividade de planejamento, induzindo à prospecção de curto prazo. Quando o horizonte de tempo é mais longo, as incertezas aumentam e a quantidade de pessoas internas da empresa que participam do processo diminui. Recorre-se a métodos que envolvam interatividade pessoal e que facilitem o debate interpessoal, daí a razão de Cenários ser mais utilizado.

\subsection{SOBRE A IMPORTÂNCIA GERAL ATRIBUÍDA À PROSPECÇÃO DO FUTURO}

A prospecção é considerada "extremamente importante" ou "muito importante" para quase 87\% dos respondentes. Este resultado corrobora o que constatou Schwarz (2008). 
A Prospecção do Futuro como Suporte à Busca de Informações para a Decisão Empresarial

Tabela 2 - Importância geral da prospecção do futuro

\begin{tabular}{|l|c|c|}
\hline IMPORTÂNCIA & FREQUÊNCIA & $\%$ \\
\hline 5: extremamente importante & 56 & 44,1 \\
\hline 4: muito importante & 54 & 42,5 \\
\hline 3: importante & 14 & 11 \\
\hline 2: muito pouco importante & 2 & 1,6 \\
\hline 1: nada importante & 1 & 0,8 \\
\hline Total & 127 & 100 \\
\hline
\end{tabular}

Fonte: Elaborado pelos autores.

\subsection{SOBRE A ASSOCIAÇÃO ENTRE MÉTODOS DE PROSPECÇÃO E A INFORMAÇÃO}

A análise da associação entre os Métodos de Prospecção e as categorias de Informação resultou, pela prova do Qui-quadrado que a hipótese de independência foi rejeitada $(\chi 2=385,9 ; \mathrm{p}=$ $0,000<0,05)$. A técnica utilizada para aprofundar a análise foi a Análise de Correspondência Simples. A Tabela 3 e o Gráfico 2 mostram, respectivamente, a variância explicada pelas dimensões e a projeção bidimensional. 
Nelson Daishiro Yoshida, James Terence Coulter Wright \& Renata Giovinazzo Spers

Tabela 3 - Percentual da variância explicada

\begin{tabular}{|c|c|c|}
\hline DIMENSÃO & \% VARIÂNCIA & $\begin{array}{c}\text { \% VARIÂNCIA } \\
\text { ACUMULADA }\end{array}$ \\
\hline 1 & $60,1 \%$ & $60,1 \%$ \\
\hline 2 & $26,1 \%$ & $86,2 \%$ \\
\hline 3 & $7,0 \%$ & $93,2 \%$ \\
\hline 4 & $3,5 \%$ & $96,7 \%$ \\
\hline 5 & $2,6 \%$ & $99,4 \%$ \\
\hline 6 & $0,4 \%$ & $99,8 \%$ \\
\hline 7 & $0,2 \%$ & $100,0 \%$ \\
\hline Total & $100,0 \%$ & $100,0 \%$ \\
\hline
\end{tabular}

Fonte: Elaborado pelos autores.

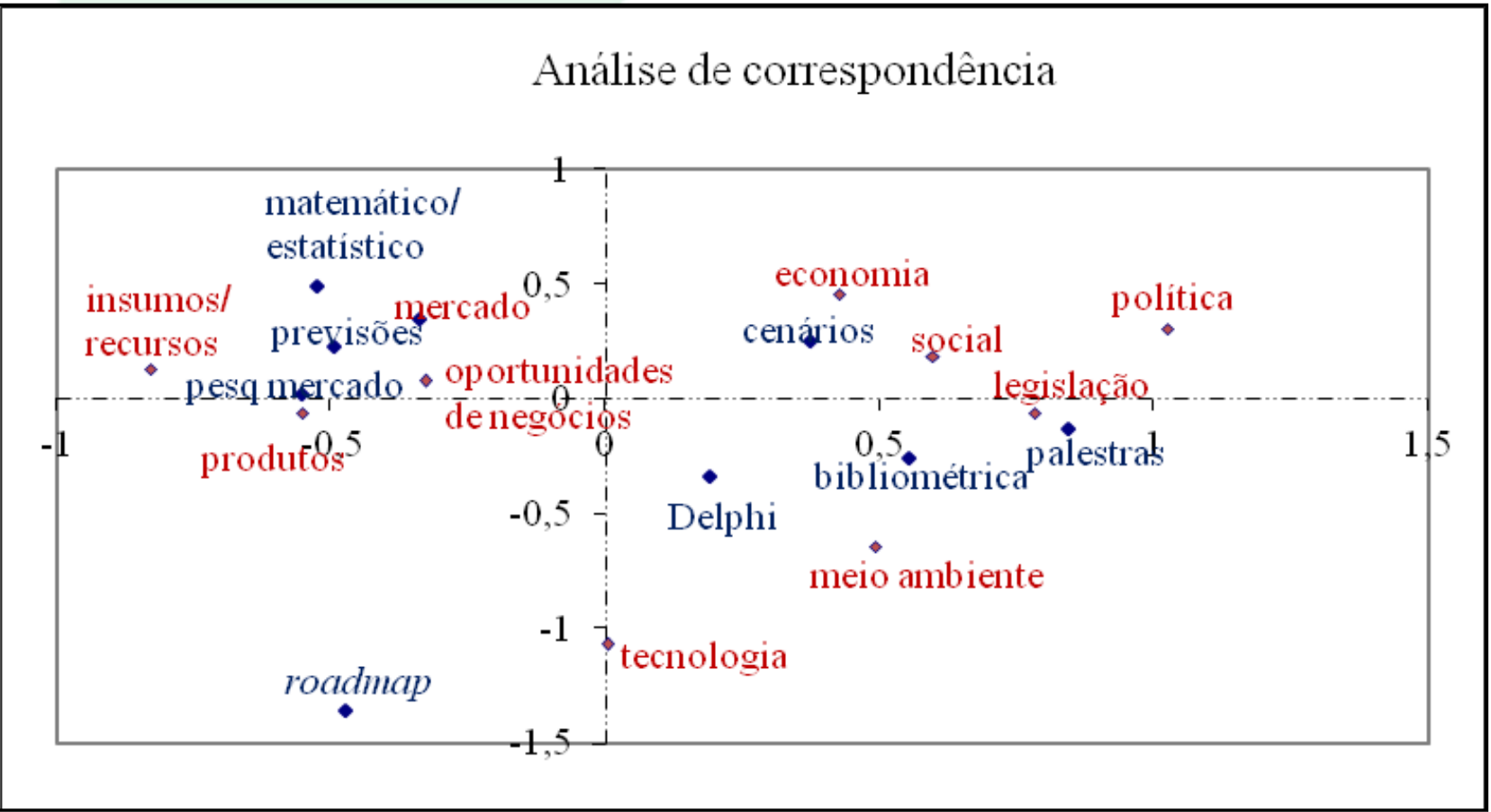

Gráfico 2 - Projeção bidimensional das variáveis Métodos de Prospecção e Informação Fonte: Elaborado pelos autores. 
A Prospecção do Futuro como Suporte à Busca de Informações para a Decisão Empresarial

No Gráfico 2, os Métodos estão grafados em azul e as categorias de Informação (temas prospectados) em vermelho. Do lado positivo do eixo horizontal, as categorias da variável Informação que ali se encontram representam temas externos à organização. Dessa forma,elas podem demandar mais esforços para mitigar incertezas, pois não são variáveis controláveis pelos gestores das empresas. Ali se encontram as categorias (de Informação): Política, Economia, Social, Legislação, Meio Ambiente e Economia.

Quanto à variável Métodos de Prospecção, no lado direito do Gráfico 2estão Cenários, Palestras de Especialistas, Análise Bibliométrica e Delphi. As categorias de métodos Cenários, Palestras de Especialistas, Análise Bibliométrica e Delphi são, dessa forma, mais adequadas para lidar com informações sobre Política, Economia, Social, Legislação e Meio Ambiente. Delphi e Análise Bibliométrica são métodos que se encontram mais distantes de Economia, Social e Política, portanto seu uso para lidar com esses temas pode não ser adequado.

No eixo horizontal negativo (lado esquerdo), as categorias das variáveis em estudo têm relação com temas internos às empresas, situação em que parte das variáveis pode ser diretamente gerenciável pelos gestores, portanto o esforço para mitigar incertezas pode ser menor do que do lado direito do Gráfico 2. Em relação à variável Informação, estão representadas nesse lado: Insumos e Recursos, Produtos, Mercado e Oportunidades de Negócios. Em relação à variável Métodos de Prospecção, ali são encontrados: Pesquisas de Mercado, Previsões e Projeções de Executivos, Força de Vendas e Distribuidores e os métodos Matemáticos. Os métodos ali encontrados podem ser adequados para lidar com as categorias de informação ali localizadas. Roadmap Tecnológico, contudo, está mais distante das categorias Insumos e Recursos, Produtos e Oportunidades de Negócios. Isso indica que o método tem associação menos forte com essas categorias, podendo apresentar alguma limitação de uso para lidar com esses temas indutores da prospecção.

A primeira dimensão (eixo horizontal) explica 60,1\% da variância total, e é denominada nesta pesquisa como fator "Mitigação de Incertezas". Ela é assim denominada porque a variável Informação apresenta duas categorias mais significantes nesse eixo, e elas estão em posições opostas no eixo. No extremo direito se encontra Política, e no esquerdo, Insumos e Recursos. O primeiro é de ambiente amplo e o segundo tem mais relação com temas mais operacionais dos negócios da empresa. A disponibilidade de informação e a sua análise contribui para lidar com incertezas, o que define o eixo horizontal como fator de "Mitigação de Incertezas".

A segunda dimensão (eixo vertical) explica 26,1\% da variância total. Neste eixo a categoria de métodos Matemáticos se encontra próximo do método Previsões e Projeções de Executivos, 
Força de Vendas e Distribuidores, e ambos estão próximos de Mercado. Portanto os métodos Matemáticos, Previsões e Projeções de Executivos, Força de Vendas e Distribuidores podem ser adequados para buscar e analisar informação da categoria Mercado.

Considerando essa segunda dimensão (eixo), do lado positivo deste eixo o método de Cenários se encontra próximo das categorias Economia e Social, que são categorias da variável Informação. Isso pode indicar maior adequação de Cenários para lidar com informações sobre economia e sociedade. Essas combinações no eixo vertical positivo podem indicar maior familiaridade para lidar com essas informações pelo método de Cenários.

Por outro lado, as categorias de informação Meio Ambiente e Tecnologia estão na parte de baixo do Gráfico 2. Como não há métodos de prospecção muito próximos, possivelmente há uma lacuna no tratamento dessas categorias de informação quando se trata de prospecção do futuro.Os métodos mais próximos para Meio Ambiente e Tecnologia são o Delphi e o Roadmap Tecnológico. As distâncias constatadas entre elas, contudo, não são pequenas, o que indica haver possível limitação para lidar com essas categorias de informação por meio desses métodos, embora eles sejam, dentre os métodos aqui estudados, os mais fortemente associados com essas categorias de informação. Esse resultado indica haver necessidade de se estudar porque isso ocorre.

Uma suposição é que a amostra de respondentes pode representar empresas que não lidam muito com prospecção de tecnologia. Outra suposição é que o nível de familiaridade com a prática dos métodos Delphi e Roadmap Tecnológico não seja bom.

Nesta pesquisa a segunda dimensão (vertical) é denominada de fator "Familiaridade". No extremo positivo vertical estão métodos Matemáticos e informações sobre Economia e Mercado. No lado negativo vertical estão métodos Roadmap Tecnológico, Delphi e Análise Bibliométrica e informações sobre Tecnologia e Meio Ambiente. Embora sejam categorias próximas entre si, as distâncias ainda são grandes, portanto são associações menos fortes. Esta é a razão pela denominação do eixo vertical como fator "Familiaridade", ela significa que categorias próximas na parte positiva representam combinações adequadas em termos de operar os métodos para buscar as categorias de informação ali encontradas. Do lado negativo, contudo, as associações são menos fortes, e mesmo havendo alguma proximidade entre categorias, ou pode haver limitação na aplicação do método ou a categoria de informação não está no radar da prospecção. Quando as distâncias entre categorias são maiores, ocorre limitação em lidar tanto com os métodos quanto com as informações.

A análise dessa diferença de força nas associações do lado superior e inferior do Gráfico 2aponta indícios de oportunidades de pesquisa. Uma primeira, com foco na teoria dos métodos e 
A Prospecção do Futuro como Suporte à Busca de Informações para a Decisão Empresarial

suas recomendações de como aplicá-la (prescrição), e uma segunda com uma abordagem focada nos temas indutores de prospecção que resultaram em associações menos fortes com os métodos.

Observam-se quatro conjuntos de agrupamentos contendo categorias das variáveis Métodos de Prospecção e Informação, conforme ilustra o Quadro 2.

\begin{tabular}{|c|c|c|}
\hline CONJUNTO DE PONTOS & CATEGORIAS DE INFORMAÇÃO & MÉTODO DE PROSPECÇÃO \\
\hline 1 & Política, Economia, Social, Legislação & Palestras e Cenários \\
\hline 2 & Legislação, Meio Ambiente, Social & Análise bibliométrica \\
\hline 3 & $\begin{array}{c}\text { Insumos e Recursos, Produtos, Mercado, } \\
\text { Oportunidades de Negócios }\end{array}$ & $\begin{array}{c}\text { Previsões e projeções de } \\
\text { executivos..., Pesquisa de } \\
\text { mercado, métodos } \\
\text { Matemáticos/Estatísticos }\end{array}$ \\
\hline 4 & Tecnologia & $\begin{array}{c}\text { Método Delphi e Roadmap } \\
\text { Tecnológico }\end{array}$ \\
\hline
\end{tabular}

Quadro 2 - Agrupamentos de Método de prospecção x Informação com associação mais forte Fonte: Análise dos autores.

Em cada conjunto de pontos descritos no Quadro 2, as categorias da variável Informação representam as que têm mais adequação para serem buscadas e analisadas pelas respectivas categorias da variável Métodos.

O conjunto de pontos 1, do Quadro 2, indica que busca e análise de informações sobre Política, Economia, Sociedade e Legislação possa ter mais propensão a ser realizada pelo uso dos métodos de Cenários e Palestras de Especialistas. Observa-se que o método de Cenários está acima do de Palestras de Especialistas, mostrando maior familiaridade com o uso deste método para lidar com as categorias de informação indicadas, isso se comparado ao uso de Palestras de Especialistas para as mesmas categorias de informação.

O conjunto de pontos 2, do Quadro 2, indica a Análise Bibliométrica como o método próximo de temas como Legislação, Sociedade e Meio Ambiente. Há, contudo, a constatação de que o método ainda carece de familiaridade no seu uso, pois ele se encontra na parte negativa do eixo vertical. Foi constatado que o método é um dos menos utilizados, e a análise de correspondência resultou que, para as categorias de informação citadas, os resultados da amostra de respondentes mostra uma possível adequação da Análise Bibliométrica para lidar com temas de 
Legislação, Sociedade e Meio Ambiente. O método Delphi poderia ser considerado para a categoria de informação Meio Ambiente, devido à proximidade entre estas categorias. Contudo, cabem as mesmas ressalvas sobre a familiaridade feitas para a Análise Bibliométrica para lidar com MeioAmbiente.

No conjunto de pontos 3, do Quadro 2, tem-se a combinação das categorias de informação sobre Insumos e Recursos, Produtos, Mercado e Oportunidades de Negócios com os métodos de Pesquisa de Mercado, Previsões e Projeções de Executivos, Força de Vendas e Distribuidores e métodos Matemáticos. Nota-se que informações sobre Produtos estão mais fortemente associados com os métodos de Pesquisa de Mercado e Previsões de Executivos. Informações sobre o Mercado e Oportunidades de Negócios estão associadas com métodos Matemáticos e Pesquisa de Mercado, mas essas categorias se encontram mais à direita se comparados com Produtos e principalmente com Insumos e Recursos. Isto é sinal de que os temas ligados a Mercado e a Oportunidades de Negócios possam demandar mais esforço dos gestores para mitigar incertezas se comparados com Produtos e Insumos e Recursos.

No conjunto de pontos 4, do Quadro 2, encontra-se a categoria de informação relacionada à Tecnologia. Os métodos que mais se aproximam dele são Delphi e Roadmap Tecnológico, mas ainda assim não estão muito próximos. Isto pode ser um sinal de que a busca e análise de informações sobre Tecnologia seja um objetivo pouco perseguido pelos respondentes da amostra, ou o uso dos respectivos métodos não seja de domínio dos respondentes, limitando sua adoção para a prospecção. Outra causa possível pode ser atribuída à natureza de inovação inerente ao tema Tecnologia, e também à menor familiaridade com os métodos Delphi e Roadmap Tecnológico, trazendo limitações no uso dos mesmos. Não se pode concluir sobre essas suposições, o conjunto de pontos 4 pode gerar temas de pesquisas futuras.

Em termos gerais, na amostra de respondentes desta pesquisa, os resultados obtidos na Análise de Correspondência Simples mostram que os fatores Mitigação de Incertezas (na horizontal) e Familiaridade (na vertical) podem ter papel importante na atividade de prospecção do futuro.

\subsection{SOBRE A SATISFAÇÃO COM A PRÁTICA DA PROSPECÇÃO}

Os resultados da amostra indicaram que Pesquisas de Mercado, Cenários e Previsões e Projeções de Executivos, Força de Vendas e de Distribuidores são os métodos que apresentam maior satisfação, com respectivamente $91 \%, 88 \%$ e $88 \%$ na soma de "Satisfeito", "Muito Satisfeito" e "Totalmente Satisfeito". Roadmap Tecnológico também teve alta satisfação (67\% nos 
A Prospecção do Futuro como Suporte à Busca de Informações para a Decisão Empresarial

mesmos itens), mas foi o segundo colocado na frequência de insatisfeitos (14\%).Esse resultado da fase quantitativa converge com as declarações obtidas nas entrevistas da fase qualitativa. Os métodos que permitem interatividade e julgamento são citados como os que mais contribuem para a tomada de decisão. Os entrevistados demonstraram preferência por interação com outras pessoas, pares da empresa, especialistas, consultores, fornecedores e distribuidores.

Os métodos Matemáticos ficaram em um nível bom de satisfação ( $80 \%$ nos mesmos itens). Contudo, nas entrevistas foi citada a necessidade de "validação" dos resultados prospectados. O termo foi referido num contexto em que um resultado de uma prospecção não tem paralelo de comparação, demandando alguma outra forma de corroborar ou criticar o resultado.

Os métodos Análise Bibliométrica e Delphi apresentaram menor satisfação(54\% e 55\% nos mesmos itens). Roadmap Tecnológico ficou em segundo lugar em termos de respondentes insatisfeitos, apesar de ter tido 42,9\% de "Totalmente Satisfeito" e "Muito Satisfeito". Nas entrevistas foi constatado que métodos que não permitem julgar e debater o futuro de forma interativa e pessoalsão considerados mais difíceis de lidar. Isso explicaria porque Roadmap obteve boa avaliação de satisfação se comparado com Análise Bibliométrica e Delphi. Estes últimos são realizados sem a possibilidade de interação entre os participantes.

Analisando-se os resultados obtidos nas fases quantitativa e qualitativa, observa-se que pode haver questões sobre confiabilidade nos resultados da prospecção do futuro. Como esta variável não foi incluída nesta pesquisa, pode ser tema de pesquisas futuras.

\subsection{SOBRE A IMPORTÂNCIA DA PROSPECÇÃO DO FUTURO E O TIPO DE DECISÃO}

Para a importância geral da prospecção do futuro, os resultados quantitativos mostram que os respondentes da amostra consideram importante a atividade de prospecção do futuro para suporte à tomada de decisão (87\% de "5 - Extremamente Importante” e “4 - Muito Importante").

Esse resultado converge com o relatado pelos entrevistados nos estudos de casos, onde foi observado que análises sobre o futuro são consideradas relevantes no processo de decisão. Em resumo, os casos estudados indicaram que a atividade de prospecção do futuro é considerada, no mínimo, uma atividade necessária para suporte à decisão.

Quando colocado o tipo de decisão na pergunta, os resultados quantitativos mostram que a amostra apresenta distribuições diferentes, e as diferenças não são casuais. Quando a decisão é do tipo estratégico, aumenta a quantidade de respostas para as categorias "5 - Extremamente Importante" e "4 - Muito Importante". Neste caso, elas somam 96\% para decisões estratégicas, 
contra $87 \%$ quando o tipo de decisão não era considerado na pergunta.Quando o tipo de decisão foi tático, a mesma soma caiu para $43 \%$. A prova de Kolmogorov-Smirnov mostrou que, ao nível de significância $\alpha=0,05$, há diferença na importância atribuída à prospecção em função da decisão envolvida ser estratégica ou tática.

\section{CONSIDERAÇÕES FINAIS}

A prática da prospecção do futuro foi constatada na amostra de executivos dessa pesquisa, sendo que os métodos utilizados e os temas indutores que levam os gestores a recorrer à atividade foram identificados. Alguns aspectos encontrados nos resultados devem ser comentados, com o objetivo de contribuir com as conclusões e apontar oportunidades de pesquisas no futuro.

A pesquisa apontou que a prática da prospecção é atividade presente e recorrente nas organizações da amostra pesquisada, e os gestores pesquisados consideram a atividade importante para o suporte à tomada de decisões. Os métodos mais praticados foram identificados, os horizontes de tempo e a frequência de uso da prospecção foram caracterizados. Os temas indutores da prospecção foram estudados e verificou-se que eles são associados aos métodos, sendo possível estimar recomendações de métodos em função do tipo de tema indutor de prospecção. A pesquisa contribuiu para identificar que algumas dessas recomendações são mais diretas, já outras, nem tanto.

Se por um lado, a atividade é uma prática valorizada nas organizações, por outro lado ela demonstra sofrer alguma limitação que merece atenção em termos de aplicabilidade nas empresas e instituições. Esta limitação pode ter componentes relacionados com a teoria disponível sobre prospecção do futuro e a natureza prescritiva do seu uso e sua disseminação nas organizações. Também cabe mencionara existência de componentes relacionados com as lacunas que podem existir, especialmente quando se lida com determinados temas indutores da prospecção e as respectivas metodologias que possam contribuir para lidar com eles.

Os resultados apontam indícios de que a teoria disponível sobre os métodos pode não conter lacunas, o que converge com a literatura, mas ao se considerar os diferentes temas indutores de prospecção, a aplicação da teoria pode necessitar de recomendações metodológicas que mitiguem as limitações em lidar com temas que não tenham tido forte associação com métodos. Esta á uma das contribuições desta pesquisa.

Seguindo com as conclusões, os resultados mostram que os gestores pesquisados utilizam mais de um método de prospecção (512 citações de métodos para 127 respondentes - da Tabela 1), 
resultando em aproximadamente 4 métodos em média. Isso pode ser um ponto positivo, pois a teoria recomenda o uso de mais de um método para lidar com mesmo problema de prospecção, contudo, observou-se nos casos estudados que os gestores tendem a preferir métodos que permitam interação entre as pessoas. Dessa forma, ocorre maior recorrência a métodos de julgamento ou de opinião, seja para se prospectar preliminarmente, seja para confirmar ou validar os resultados obtidos em uma primeira análise realizada. A combinação de métodos de julgamento com outros de natureza mais estruturada, que se constitui numa recomendação prescritiva da teoria, pode não estar ocorrendo.

Uma oportunidade de pesquisa futura, portanto, decorre dessa constatação, uma vez que nesta pesquisa não foi foco central o estudo de uso combinado de métodos para um mesmo problema de prospecção. Adicionalmente, se os gestores não sentem falta de métodos prospectivos disponíveis, a sua prática pode não estar cobrindo todas as possibilidades de benefícios, uma vez que métodos objetivos e estruturados, usados em combinação com os métodos de julgamento, podem facilitar as análises prospectivas.

Este estudo carrega limitações, especialmente com relação à amostra utilizada na pesquisa de campo e a impossibilidade de generalizar os resultados. Ainda assim, este trabalho contribui significativamente para o entendimento sobre a utilização de métodos prospectivos nas organizações, apresentando informações relevantes para os tomadores de decisão nas organizações, com relação à utilização de métodos e abordagens de prospecção, assim como apresenta resultados exploratórios interessantes para a Academia, que poderão ser aprofundados em pesquisas futuras, conforme mencionado anteriormente. Destaca-se que foi identificado que há associação entre a categoria da informação prospectada e os métodos. Os métodos mais praticados são os que apresentam a possibilidade de interatividade pessoal, sendo que métodos objetivos, como os que utilizam equações, não figuraram entre os mais praticados. Há uma preocupação sobre a necessidade de estruturação da atividade de prospecção nas empresas e sobre a prática da mesma seguindo recomendações metodológicas. Estes resultados demonstram uma clara relação entre os avanços metodológicos que podem ser consolidados e disseminados pela Academia e a prática das organizações em prospecção. 


\section{REFERÊNCIAS}

Bioagência (2011) www.bioagencia.com.br acesso em abril de 2011.

Bolger, Fergus; Wright, George (1994).Assessing the quality of expert judgment.DecisionSupport Systems 11 (1994) 1-24.

Brasil Tratado de Itaipu (1973)Brasília,26.4.1973.Disponívelem http://www2.itaipu.gov.br/empre/docum_trata.htm\#top acessado em abril/2011.

CTC-CentrodeTecnologiaCanavieira (2011).Acessopelo endereço http://www.ctcanavieira.com.br/site/index.php acessado em abril de 2011.

Chermack, Thomas J. (2006)Assessing the quality of scenarios in scenario planning. FuturesResearchQuarterly, Winter 2006.

Creswell. John W. (2010) Projeto de pesquisa: métodos qualitativo, quantitativo e misto - $3^{\text {a }}$ Ed. Porto Alegre: Artmed, 2010.

Daim, Tugrul U.; Ploykitikoon, Pattravadee; Kennedy, Elizabeth; Choothian, Woraruthai (2008)Forecasting the future of data storage: case of hard disk drive and flash memory.Foresight, vol. 10, issue 5, 2008.

Day, George S.; Schoemaker, Paul J.H.(2004)Driving Through the Fog: Managing at the Edge. Long Range Planning 37 (2004) 127-142.

Dawes, Robyn M. (1979) The robust beauty of improper linear models in decision making. American Psychologist, Vol. 34, N 7, 571-582, July,1979.

Eisenhardt, Kathleen M.; Zbaracki, Mark J. (1992)Strategic Decision Making.Strategic Management Journal, Vol. 13, 17-37 (1992).

Eisenhardt, Kathleen M. (1989)Building theories from case study research. The Academy of Management Review, Vol. 14, No. 4. (Oct., 1989), pp. 532-550.

Fildes, Robert; Goodwin, Paul (2007)Against Your Better Judgment? How Organizations Can Improve Their Use of Management Judgement in Forecasting. Interfaces; Nov/Dec 2007; 37, 6.

Galbraith, Jay (1974)Organization Design: an information processing view. Interfaces, vol. 4, $\mathrm{n}^{\circ} 3$, may, 1974.

Georgoff, David M.; Murdick, Robert G. (1986)Manager's guide to forecasting.Harvard Business Review, 1986. 
Godet, Michel; Roubelat, Fabrice (1996)Creating the future: the use and misuse of scenarios.Long Range Planning, Vol. 29, No. 2, pp. 164 to 171, 1996.

Hogarth, Robin M.; Makridakis, Spyros (1981)Forecasting and planning: an evaluation.Management Science (pre-1986); Feb 1981; 27, 2.

ItaipuBinacional (2009) Itaipu:usinahidrelétrica-projeto:aspectosdeengenharia.. Foz do Iguaçu: TAB Marketing Editorial, 2009.

Kaplan, Robert S.; Norton, David P. (2008)The execution premium: linking strategy to operations for competitive advantage. Harvard Business School Publishing, Boston, Massachusetts, 2008.

Kotler, Philip; Keller, Kevin L. (2006)Administração de marketing 12a ed. São Paulo: Pearson Prentice Hall, 2006.

Lee, Sungjoo; Park, Yongtae (2005)Customization of technology roadmaps according to roadmapping purposes: Overall process and detailed modules. Technological Forecasting \& Social Change 72 (2005) 567-583.

Mahmoud, Essam (1989)Combining forecasts: Some managerial issues. International Journal of Forecasting 5 (1989) 599-600.

Makridakis, Spyros; Hogarth, Robin M.; Gaba, Anil (2009)Forecasting and uncertainty in the economic and business world. International Journal of Forecasting 25 (2009) 794-812.

Martino, Joseph P. (1993) Technological forecasting for decision making. New York: McGraw-Hill c1993.

Mentzler, John T.; Kahn, Kenneth B. (1995)Forecasting technique familiarity, satisfaction, usage, and application.Journal of Forecasting, Vol. 14, 465-476 (1995).

Milliken, Frances J. (1987)Three Types of Perceived Uncertainty about the Environment: State, Effect, and Response Uncertainty. The Academy of Management Review, Vol. 12, No. 1 (Jan., 1987), pp. 133-143.

Phaal, Robert; Farrukh, ClareJ.P.; Probert, DavidR. (2004) Technology roadmapping - A planning framework for evolution and revolution.Technological Forecasting \& Social Change 71 (2004) $5-26$.

Phlips, Louis. (1988)The economics of imperfect information. New York - Cambridge University Press, 1988.

Porter, Alan L.; Roper, A. Thomas.; Mason, Thomas H.; Rossini, Frederick H.; Banks, Jerry (1991)Forecasting and Management of Technology. John Wiley \& Sons, 1991. 
Porter, Alan L. (2007)How "tech mining" can enhance r\&d management. Research Technology Management; Mar/Apr 2007; 50, 2, pg. 15.

Rowe, Gene; Wright, George (1999)The Delphi technique as a forecasting tool: issues and analysis. InternationalJournalofForecasting 15 (1999) 353-375.

Sampieri,RobertoH.;Collado,CarlosF.;Lucio,PilarB.(2006) Metodologiade Pesquisa. 3. ed. São Paulo: McGraw-Hill, 2006.

Sardar, Ziauddin (2010)The Namesake: Futures; futures studies; futurology; futuristic; foresightWhat's in a name? Futures 42 (2010) 177-184.

Schoemaker, Paul J. H. (1995) Scenario Planning: A Tool for Strategic Thinking.Sloan Management Review; Winter 1995; 36, 2.

Schwarz, Jan O. (2008)Assessing the future of futures studies in management. Futures 40 (2008) 237-246.

Simon, Herbert A. (1997)Administrative behavior.A study of decision-making in administrative organizations - 4th edition. The Free Press, New York, 1997.

Strand, Sverre (1999)Forecasting the future: pitfalls in controlling for uncertainty.Futures 31 (1999) $333-350$.

Vidican, Georgeta; Woon, Wei L.; Madnick, Stuart (2009)Measuring innovation using bibliometrictechiques: The case of solar photovoltaic industry.Sloan School of Management, MIT, 2009.

Walsh, Philip R. (2005) Dealing with the uncertainties of environmental change by adding scenario planning to the strategy reformulation equation. Management Decision Vol. 43 No. 1, 2005 pp. 113-122.

Winklhofer, Heidi; Diamantopoulos, Adamantios; Witt, Stephen F. (1996) Forecasting practice: a review of the empirical literature and an agenda for future research. InternationalJournalofForecasting 12 (1996) 193-221.

Wright, James T.C.; Giovinazzo, Renata A. (2000 Delphi- Umaferramentadeapoio aoplanejamentoprospectivo. CadernodePesquisasemAdministração,SãoPaulo, v.01, $\mathrm{n}^{\circ} 12,2^{\circ}$ $\operatorname{trim} / 2000$.

Wright, James T.C.; Spers, Renata A.G. (2006) O país no futuro: aspectos metodológicos e cenários. Estudos Avançados 20 (56), 2006. 
Recebido: $28 / 11 / 2012$

Aprovado: 25/01/2013 